\title{
Anomalous diffusion of hydrogen in fused quartz glasses for lamps above $800^{\circ} \mathrm{C}$
}

\author{
Kazuyuki OGAWA* Tsutomu MORIWAKI* \\ Hitoshi IMAMURA* and Yoshimi AKA *
}

\begin{abstract}
To clarify the mechanism of hydrogen diffusion in fused quartz at elevated temperatures over $800^{\circ} \mathrm{C}$, a mass filter was used to measure diffusion coefficients, permeabilities and solubities of hydrogen in several types of fused quartz tubes. The diffusion coefficient obtained at $800^{\circ} \mathrm{C}$ was $2-3 \times 10^{-6} \mathrm{~cm}^{2} / \mathrm{sec}$, and the activation energy of the hydrogen diffiusion at $400^{\circ}-800^{\circ} \mathrm{C}$ was $11.6 \mathrm{Kcal} / \mathrm{mol}$. These values are in good agreement with the values previously reported. Precise measurements at tempera. tures abobe $800^{\circ} \mathrm{C}$ resulted in diffusion anomaly, or an unusual fall-oiff of diffusion coeffusion coefficient, while premeabilities kept monotonous increase with rising temperature. In consequence the solubilities, obtained from the coefficient and permeability, showed a sharp increase up to $2 \times 10^{19}$ molecules $/ \mathrm{cm}^{3}$.
\end{abstract}

\section{Introduction}

Quartz glasses are widely used for the newly developed various lamps, because of their superiorities in transparency, chemical stability, kinetic strength and manufacturing. However, it is well known that light molecules as hydrogen, helium and so on permeate easily through them. Especially for high pressure discharge lamps, intrusion of hydrogen into discharge space affects seriously on the starting voltage of the discharge $e^{1)-3)}$. The behavior of the hydrogen diffusion in fused quartz above $800^{\circ} \mathrm{C}$, which is often belongs to the temperature of the operating lamp tube, has been still ambiguous". To clarify this phenomenon, we have measured the temperature dependence of hydrogen diffusion coefficient, permeability and solubility for the fused quartz tubes which are used for new lamps. As the results, we obtained monotonously increasing diffusion coefficients with temperature below $800^{\circ} \mathrm{C}$ which are in good agreement with other papers ${ }^{13-i)}$, however diffusion coefficients abruptly fell off above $800^{\circ} \mathrm{C}$. So we can obtain a sharply increasing solubility above $800^{\circ} \mathrm{C}$ on the basis of numerical calculation from the values of the diffusion coefficient and the permeability.

* Toshiba Research and Development Center

Saiwai-ku Komukai Toshiba-cho 1, Kawasaki 210

Tokyo Shibaura Electric Co., Ltd. Lamp Division Saiwai-ku Horikawa-cho 72, Kawasalki 210

\section{The modeling of hydrogen in rused quartz}

It needs for us to make a model of the hydrogen in fused quartz corresponding the measured values to the theoretical equation in order to estimate the diffusion, permeation and solubility through/in the fused quartz.

\subsection{Modeling of the difusion}

Two vacuum chambers are partitioned by the membrane of fused quartz glass as shown in Fig. 1. One part is filled with hydrogen gas and the other is pumped out by a vacuum pump.

In this case, hydrogen molecules permeate through the glass plate and are evolved into the chamber

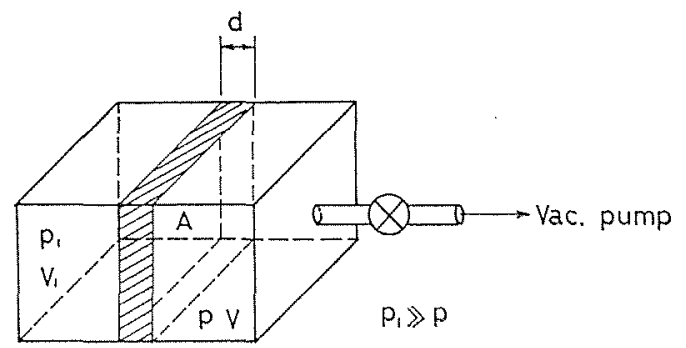

Fig. 1 Amodel of the diffusion experinent.

A: Area of the specimen

$d:$ Thickness of the specimen

$p_{1}, V_{1}$ : Pressure, volume in the higher pressure chamber

$D, V:$ Pressure, volume in the lower pressure chamber 
beong pumped out to vary the partial pressure of the hydrogen. It is assumed that the transported quantity of the hydrogen in the glass is equal to the variation of the hydrogen pressure in the output side chamber, or the law of conservation of flux is valid. Diffusion coefficient of hydrogen in fused quartz would be able to be defined under the assumption of Fick's law or diffusion law. It is proper for us to consider this case as one dimensional model, then the equation

and

$$
j=-D \partial C(x, t) / \partial x
$$

$$
\partial C(x, t) / \partial t=D \partial^{2} C(x, t) / \partial x^{2}
$$

should hold, where $j$ is the rate of the gas flow per unit area of the glass, $C$ is the concentration of the diffusion space, $x$ is the distance in the direction of the normal line for the glass surface. It is pressumed that the glass specimen has no temperature gradient. In this diffusion phenomenon as mentioned above, a basic relationship

$$
P=D S
$$

should be kept, among permeability $P$ ( Number of [molecules] - Glass thickness [cm]/Glass surface area $\left[\mathrm{cm}^{2}\right]$. Time [sec]), diffusion coefficient $D$ and solubility $S$ ( Number of [molecules]/Glass volume $\left[\mathrm{cm}^{3}\right]$ ).

\subsection{The solution of a diffusion equation.}

By corresponding the reading of the massfilter hydrogen peak to the solution of the diffusion equation, diffusion coefficient by decay method can be measured. Hydrogen gas is loaded at $1 \mathrm{~atm}$ from the outer tube, and when the steady flow of hydrogen through the glass is attained, hydrogen gas in the outer tube is quickly pumped out at time $t_{c}$. Then the time variation after $t_{c}$ of effusing hydrogen from the hydrogen loaded will be as follows ${ }^{\text {s) }}$,

$$
\begin{array}{r}
h=h_{0}\left\{2 \sum_{n=1}^{\infty}(-1)^{n+1} \exp \left[-\left(\pi^{2} n^{2} / d^{2}\right) D t\right]\right\}, \\
n=0,1,2, \ldots \ldots \ldots \ldots \ldots \ldots \ldots \ldots \ldots \ldots \ldots \ldots \ldots \ldots \ldots \ldots \ldots \ldots
\end{array}
$$

where $h$ is the reading of the hydrogen pressure, $h_{0}$ is a constant, $d$ is the wall thickness of a fused quartz specimen, $D$ is the diffusion coefficient, and $t$ is time. This typical variation of $h$ is shown in the curve after the time $t_{0}$ in Fig. 2. For enough large $t$, the progression of $n=2,3, \ldots$ could be negletced, so we obtain

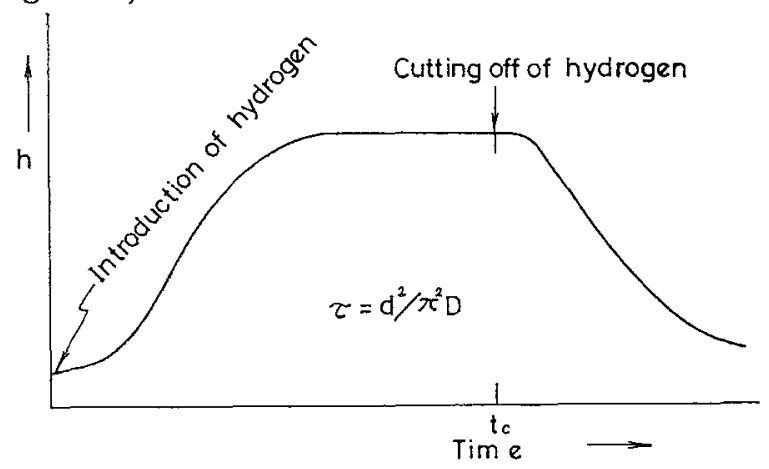

Fig. 2 Time variation of the massfilter reading of the hydrogen by flow method.

$$
h=2 h_{0} \exp \left(-\frac{\pi^{2}}{d^{2}} D t\right)
$$

The $D$ is determined from following equation

$$
D=\frac{\ln \left(h_{1} / h_{2}\right)}{\left(\pi^{2} / d^{2}\right)\left(t_{2}-t_{1}\right)},
$$

where $h_{1}$ and $h_{2}$ are the reading of hydrogen peak height of the mass spectrum corresponding to time $t_{1}$ and $t_{2}$ respectively.

In the next place, build-up method can be also used to determine these coefficients. In this case, those are determined by the increasing rate of the hydrogen pressure in closed chamber which is evacuated at first and then isolated from the pump by cut-off cock. Hydrogen partial pressure is represented as follows ${ }^{\text {) }}$,

$$
\begin{aligned}
p= & \frac{A D C}{V d} p_{1}\left\{t-\frac{d^{2}}{6 D}-\frac{2 d^{2}}{\pi^{2} D} \cdot\right. \\
& \left.\sum_{n=1}^{\infty}(-1)^{n} \frac{\exp \left(-n^{2} \pi^{2} D t / d^{2}\right)}{n^{2}}\right\}
\end{aligned}
$$

where $p$ is the hydrogen pressure in the vacuum chamber into which the hydrogen molecule flow, $p_{i}$ is set in $1 \mathrm{~atm}$ in this experiment, $A$ is the effective area of the specimen tube, $C_{1}$ is the hydrogen concentration of the outer surface of the specimen (which is assumed to be equal to the solubility of hydrogen in fused quartz), $V$ is the volume of the hydrogen out-put side chamber, $d$ is the thickness of the tubular specimen, $D$ is the diffusion coefficient of hydrogen in the specimen, and $t$ is time.

If the hydrogen permeation rate reaches the steady state, the time variation of the permeation is not affected by the progression terms in equation (7). Then equation ( 7 ) would be represented as follows,

$$
p=\frac{A D C_{1}}{V_{a}} p_{1}\left\{t-\frac{d^{2}}{6 D}\right\} .
$$

This equation show the linear portion of the curve in Fig. 3. When we let the intersection of the extention of this linear line portion and vertical be $t_{0}$, then

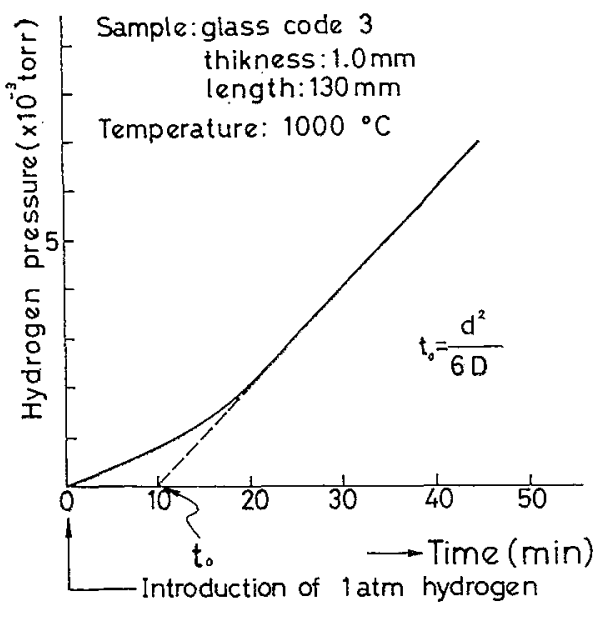

Fig. 3 Time variation of hydrogen partial pressure by build up method. 


$$
t_{0}=\frac{d^{2}}{6 D}
$$

is obtained from equation ( 8 ). $D$ can be also determined by the experimental measurement of time to. Permeability is obtained by differentiating equation (8) with respect to time $t$ as following,

$$
P=D C_{1}=\frac{V_{d}}{A p_{1}} \frac{d p}{d t}
$$

Solubility is calculated from the measure values of $P$ and $D$ using equation (3).

In this work, $D$ is obtained from both ways of equation (6) and (9), and there is a good agreement in the results by two methods.

Theoretical equation as described above is obtained about a parallel flat board specimen. In this case of the tubular diffusion specimens of this experiment, it is needed to use the reduced area of the cylindrical specimen which is closed at one end with average radius of $d=\sqrt{d_{1} d_{2}}$, where $d_{1}$ in inner and $d_{2}$ is the outer radius of the cylindrical specimen ${ }^{0}$.

\section{Experimental set-up}

The experimental set-up is shown in Fig. 4. The apparatus is made of stainless-steel except for the

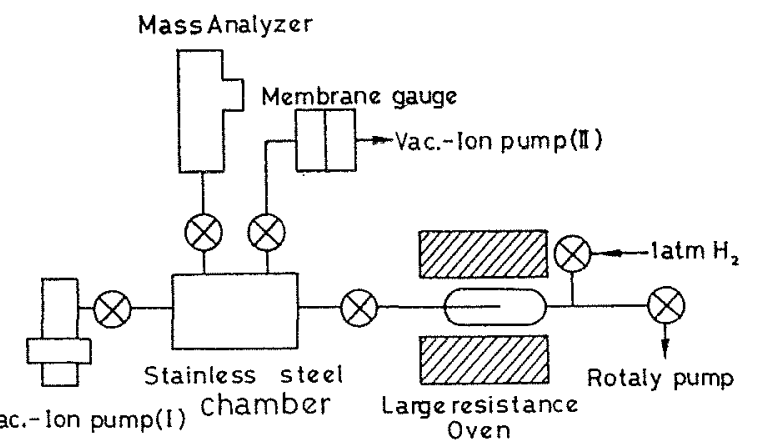

Fig. 4 Block diagram of experimental set-up used for the hydrogen diffusion measurement.

specimen glass tube portion, and baked out in vacuum at $200^{\circ} \mathrm{C}$. The attained vacuum pressure of the system is $5 \times 10^{-8}$ torr. Any temperature from room temperature to $1200^{\circ} \mathrm{C}$ was attained within the accuracy of the measurement $\pm 2^{\circ} \mathrm{C}$ by a large electric resistant oven and a temperature controller. The sputter ion pump of pumping speed $40 \mathrm{l} / \mathrm{sec}$ was used in this experiment. The mass-spectrometer for this experiment was a quadrupole type residual gas analyzer of the mass-sweep range 1 300. BA type vacuum gauge and MKS Baratron pressure meter were used in the measurement of the total pressure of this system.

\section{Specimens}

Glass specimens are arbitrarily extracted from each of several kinds of fused quartz which are all nautral fused glass in this work. These quartz tubes are fabricated in the cross-sectional form as in

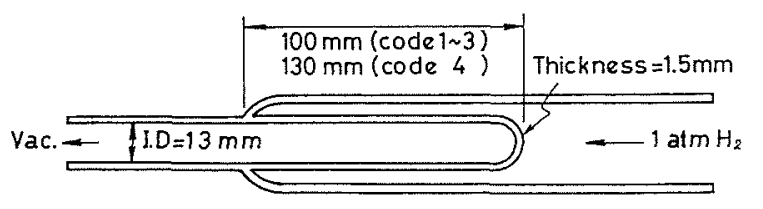

Fig. 5 Cross-sectional diagram of the cylindrical sample.

Table 1 Impurities in the fused quartz tested

\begin{tabular}{c|c|c|c|c|c|c}
\hline \multirow{2}{*}{$\begin{array}{c}\text { Sample } \\
\text { Glass Code }\end{array}$} & Type & \multicolumn{5}{|c}{ Impurities (ppm) } \\
\cline { 3 - 7 } & & $\mathrm{Al}_{2} \mathrm{O}_{3}$ & $\mathrm{Fe}_{2} \mathrm{O}_{3}$ & $\mathrm{Na}_{2} \mathrm{O}_{3}$ & $\mathrm{~K}_{2} \mathrm{O}$ & $\mathrm{TiO}_{2}$ \\
\hline 1 & $\mathrm{Vac}-$ melt & 40 & 7 & 4 & 3 & 1 \\
2 & $\mathrm{O}_{2}-\mathrm{H}_{2}$ melt & 70 & 2 & 3 & 3 & 3 \\
3 & $\mathrm{Vac}-$ melt & 65 & 3 & 4 & 3 & 3 \\
4 & $\prime \prime$ & 45 & 3 & 2 & 2 & 2 \\
\hline
\end{tabular}

Fig. 5, and then, they are dipped into $5 \% \mathrm{HF}$ liquid to eliminate the impurities on their surface. Table 1 shows the concentration of the impurities containing in each types of the samples.

\section{Results ${ }^{10}$}

\subsection{Diffusion coefficient}

Fig. 6 is the Arrhenius plot of the diffusion coefficient obtained from the experimental results using equation (6). The result shows the single Arrhenius type diffusion in each of the type in the temperature range of $400 \sim 800^{\circ} \mathrm{C}$. That is, Arrhenius equation

$$
D=D_{0} \exp (-E / R T)
$$

represents the temperature dependence of the diffusion in the temperature range, where $E$ is th activation nergy of the diffusion, $R$ is gas constant, $D_{0}$ is a constant, $D$ is the diffusion coefficient and $T$ is the absolute temperature. The activation energy calculated from the gradient of the curve in Fig. 6 is $11.6 \mathrm{Kcal} / \mathrm{mol}$ which is in good agreement with other reported values below $800^{\circ} \mathrm{C}^{4)-i(1)}$. In this work

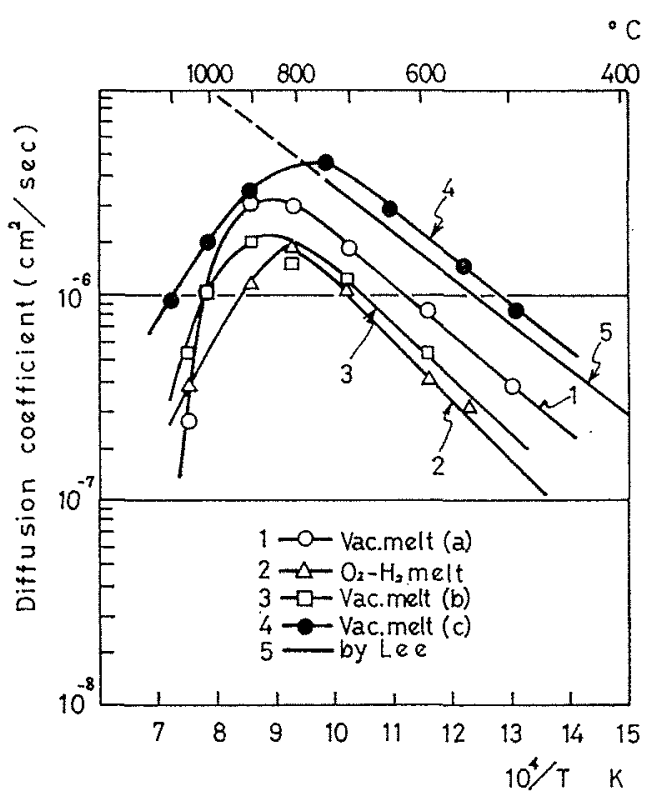

Fig. 6 Hydrogen diffusion coefficient in fused quartz. 


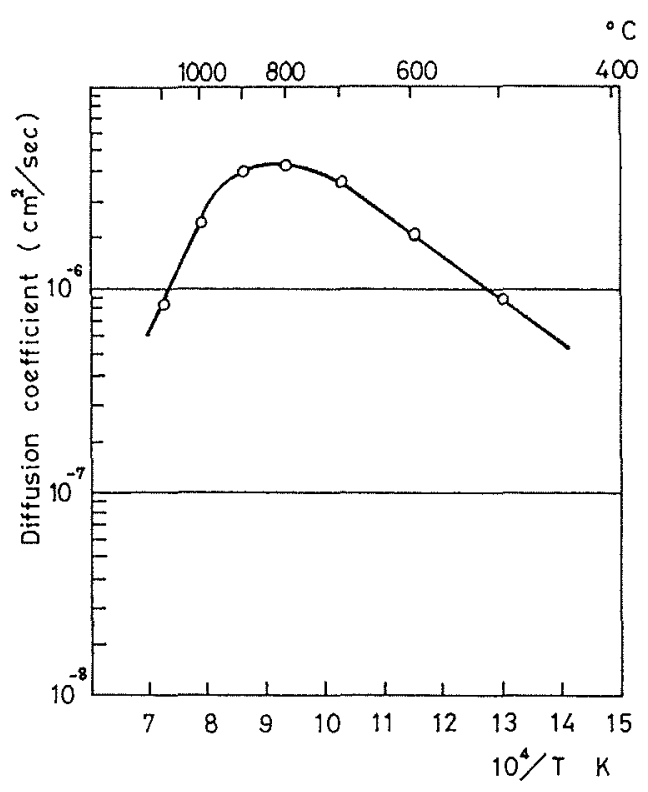

Fig. 7 Hydrogen diffusion coefficient in fused quartz (code 3) by build-up method.

above $800^{\circ} \mathrm{C}$, the diffusion coefficient shows a sharp fall-off namely non-Arrhenius or anomalous diffusion.

The above are the characteristics of the diffusion coefficient by using equation (6). Fig. 7 shows the result of the temperature dependence of the diffusion coefficient using equation (9), and shows a same tendency as Fig. 6 substantially.

\subsection{Permeability}

The MKS Baratron pressure meter was used to measure the variation of the pressure in the hydrogen out-put side chamber in the experiment to determine the permeability using equation (10). An ionization type gauge as BA gauge is not suitable for measuring the pressure of the chamber, because the pressure of the hydrogen out-put side chamber

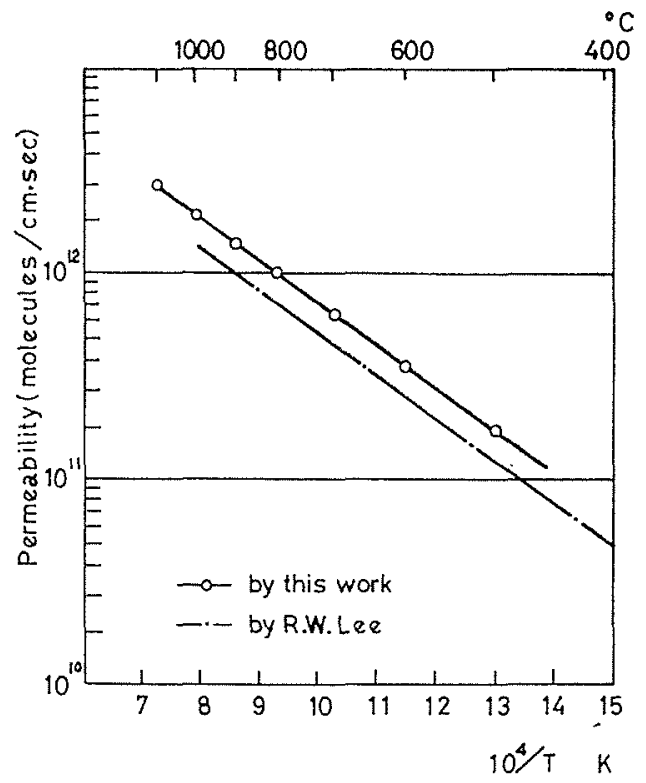

Fig. 8 Hydrogen permeability in fused quartz. is considerably high when the steady flow through the glass is attained to confirm the accurate linearity of the curve in Fig. 3. The permeabilities obtained are the values in the steady state and their Arrhenius plots of each type of the fused quartz show a good linearity in the temperature range of $400^{\circ} \sim$ $1,100^{\circ} \mathrm{C}$. This result well agrees with Lee et $\mathrm{al}^{1} \mathrm{~s}^{4) 5}$. Fig. 8 shows each Arrhenius plot of this work and Lee et al's.

\subsection{Solubility}

Fig. 9 shows the curvatures of the solubilities calculated, by using equation (3), from the diffusion coefficients and permeabilities obtained as shown in 5.1 and 5.2 .

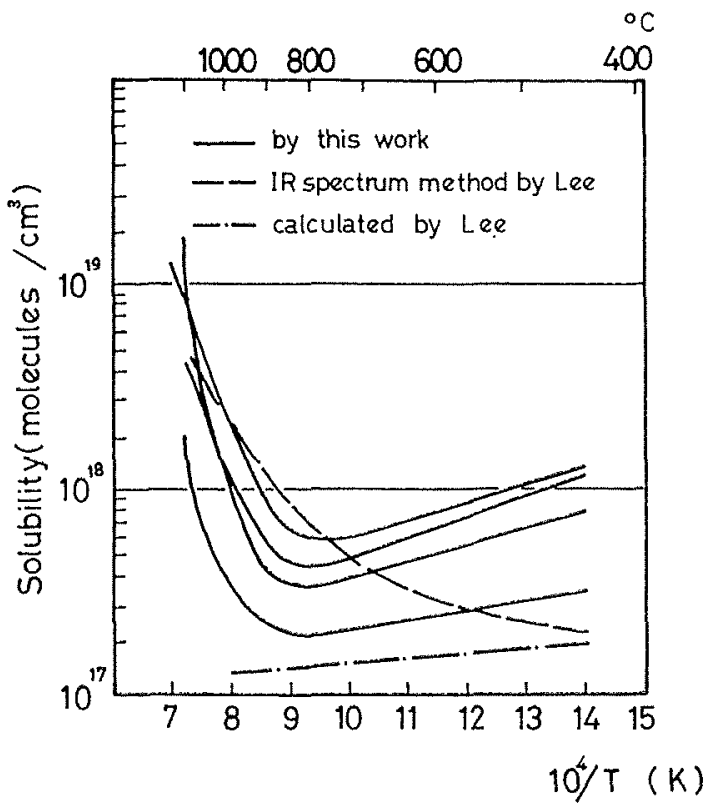

Fig. 9 Hydrogen solubility in fused quartz

It should be noted that the each solubility curve above $800^{\circ} \mathrm{C}$ shows the sharp increase with temperature corresponding with the anomalous diffusion. As the reference, the data of the solubility by the other direct measurement of Lee et al's are drawn in the same figure ${ }^{4}$.

\section{Evalution of the errors}

It is important to evaluate the errors as we analyze the data of this high vacuum experiment. The main causes of the errors are as follows ${ }^{10}$.

(a) Detector's stability and analyzing of data

(a-1) Corrected values of hydrogen partial pressure (including the stability of the detectors)

(a-2) Transformation of hydrogen to other molecules (Analyzing the mass spectrum)

(b) Conditions of the specimens

(b-1) Size of the specimen

(b-2) Stability of the measuring temperature

(c) Conditions of the vacuum system

(c-1) Stability of the exhausting speed 
(c-2) Effect of hydrogen adsorption on the wall of the vacuum system.

The results in this work will be evaluated as follows, from the stand points of the above sources of errors.

There are two methods for determining the diffusion coefficient as shown in 2.2. An exponential curve fitting is used in the determinating the diffusion coefficient from equation (6), while a linear curve fitting from the equation (9), so the latter value is naturally more accurate than the former. However, actually, we mainly used the former method, because the diffusion coefficient can be determined by the relative decay rate of the hydrogen massfilter peak reading, eliminating the error of (a-1), and the steady state of the hydrogen flow is more easily confirmed in the former than the latter. After all the measurements using the equation (6) can be done considering only the error source of $(a-1)$.

The source of (a-2) means the transformation of the hydrogen molecules to the hydrogen atom, hydroxyl radical, and the molecules including hydrogen atom as the water. By checking mass spectrum data no changes was observed throughout this experiment. Fig. 10 show the mass spectrums in the two cases of the temperature $850^{\circ} \mathrm{C}$ of the normal diffusion (a) and $1,100^{\circ} \mathrm{C}$ of the anomalous (b) respectively as the both have the same decay rate 0.55 .

In the case of the former method of the diffusion coefficient, the cause (c-1) would not affect on the measured value by nature, and the (c-2) is hardly considered to have the effect on it when the measurement is kept at the order $10^{-6}$ torr vacuum. The remaining causes are the $(b-1)$ and $(b-2)$, which affect also on other measurements in the same time. The cause (b) may affect rather on the diffusion activation energy $E$ than the constant $D_{0}$. The speci-

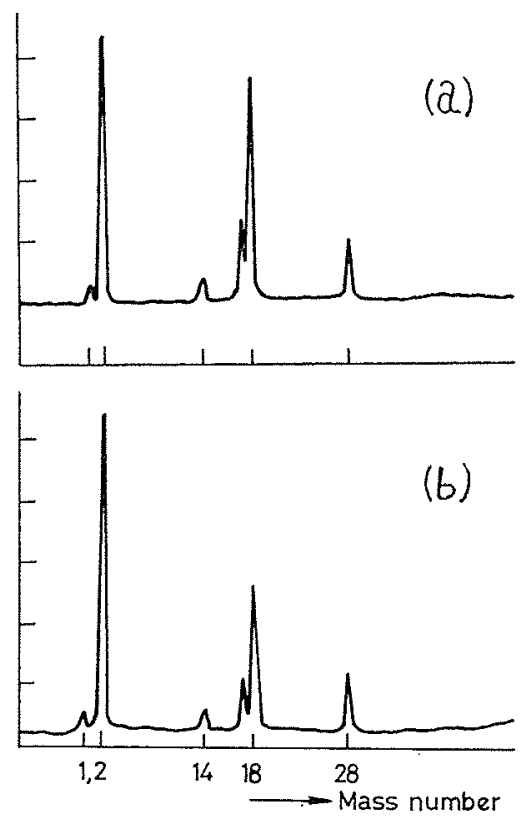

Fig. 10 Massfilter trace after decay Glass code 2

Total pressure $1.5 \times 10^{-6}$ torr (a) $850^{\circ} \mathrm{C},\left(\right.$ b) $1100^{\circ} \mathrm{C}$ men of the glass code 4 with the larger effective surface area has a larger value of $D_{0}$ which is close to Lee's. The temperature variation with the position in the oven was within $\pm 5^{\circ} \mathrm{C}$, and this might vary the value of $D_{0}$ in some degree.

In the next place, the error of the permeability will be evaluated. This measurement may include the larger error because of its nature of the absolute value measurement. It is needed for us to measure the time variation of the hydrogen pressure of the closed chamber of the out-put side which is in vacuum. So we must consider the cause (b) and (a-1) as the error the permeability and need the carefully controlled experiment. Yet the permeabilities obtained from this experiment are in good agreement with Lee's ${ }^{455}$.

The solubility $S$ in this work is the amount of permeability $P$ divided by the diffusion coefficient, therefore it may contain the both errors of the two in the same time. Then the accuracy of the solubility may be somewhat inferior to other two.

In the end, the degree of the accuracy of the three would be descrived as $D \rightarrow P \rightarrow S$ in sequence.

\section{Discussion}

On the diffusion coefficient of hydrogen in fused quartz, only a little data has been available in the temperature range exceeding about $700^{\circ} \mathrm{C}$. Moreover, these have ambiguous or scattering points in this temperature range. This work confirmed the fall off of the diffusion coefficient above $800^{\circ} \mathrm{C}$ in relation to the sharp increase of solubility with the rising temperature. A discussion will be shown as follows on a qualitative explanation of the plausible mechanism of the anomalous diffusion. As shown in Fig. 6, hydrogen diffusion coefficients in fused quartz over $800^{\circ} \mathrm{C}$ decrease with rising tempelature. This anomalous diffusion over $800^{\circ} \mathrm{C}$ may show a transition state in transfer of a recation $A$ to another $A^{\prime}$, where the two would be considered to be causing simultaniously, being mixed in a ratio varying with the temperature over $800^{\circ} \mathrm{C}$ to $1,100^{\circ} \mathrm{C}$. Reaction $A$ is the diffusion of interstitial hydrogen molecules as in Fig. 6 below $800^{\circ} \mathrm{C}$, and reaction $A^{\prime}$ is possibly diffusion of lower rates which correspond with the hopping of desociated atomic hydrogen.

Possible more precise mechanism of reaction $A$ and $A^{\prime}$ will be described as follows, using each energy model which corresponds with the molecular diffusion and, possibly, desociated hydrogen atomic diffusion, respectively. In 'channel A' of Fig. 11, a is the state of hydrogen physical adsorption on the quartz surface, whose quantity decreases with rising temperature, as is well known or as known also from the regative value of the gradient of solubility curve obtained below $800^{\circ} \mathrm{C}$ in Fig. 9. This adsorption has been investigated and found to be exothermic reaction with enthalpy of $-1.49 \mathrm{Kcal} / \mathrm{mol}^{12)}$, which shows good agreement with the value obtained from Fig. 9 below $800^{\circ} \mathrm{C}$. A transition of $\mathbf{a}$ to $\mathrm{b}$ would correspond with ramdom hopping of hydrogen molecules. The activation energy of hydrogen dif- 


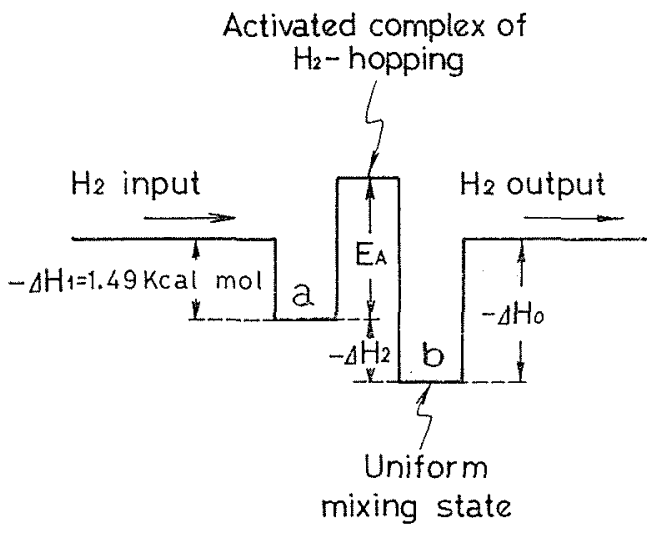

Channel A

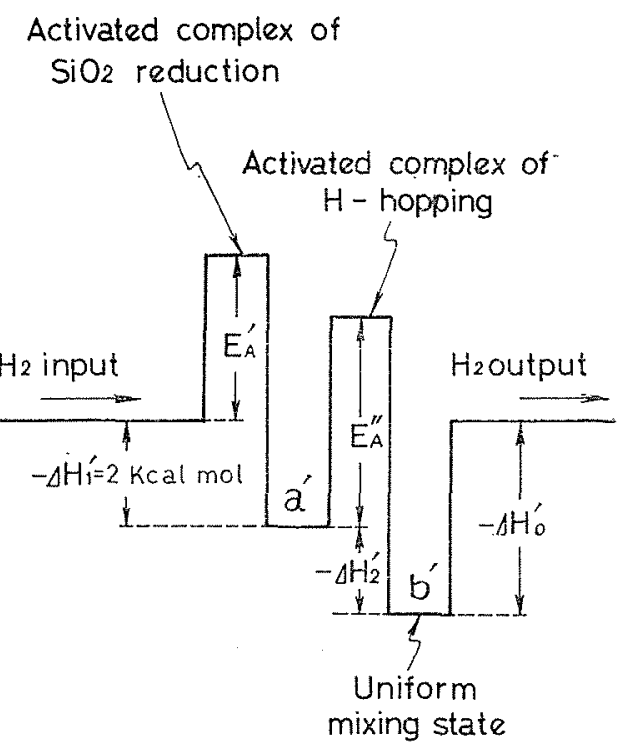

Channel Á

Fig. 11 Energy models for the two channels of hydrogen in fused quartz.

fusion $E_{A}$, which is measured from the level of a state, might be about $13 \mathrm{Kcal} / \mathrm{mol}$ as the sum of the observed value $11.6 \mathrm{Kcal} / \mathrm{mol}$ and the heat of adsorption $\left(-\Delta H_{1}\right) 1.49 \mathrm{Kcal} / \mathrm{mol}$. Energy gap $\Delta H_{2}$ might be caused mainly by hydrogen concentration difference for the distance from the quartz surface. The following refer's to "channel $A$ " which is assumed to correspond with the diffusion of atomic hydrogen in fused quartz. It is assumed that a reduction reaction of fused quartz by hydorgen

$$
\mathrm{S}_{i}{ }^{4+2} \mathrm{O}^{2-}+\frac{1}{2} \mathrm{H}_{2} \longrightarrow \mathrm{S}_{i}{ }^{3+} \mathrm{O}^{2-} \mathrm{OH}^{-} \cdots \cdots \cdots \cdots \cdots(12)
$$

is caused on the surface of the fused quartz. It is known that this reaction is exothermic and the value of the enthalpy is $-2 \mathrm{Kcal} / \mathrm{mol}^{12)}$ This reduction state will be that of $\mathbf{a}^{\prime}$ of 'channel $A$ " in Fig. 11. Energy gap $\Delta H^{\prime}$ ' between $\mathbf{a}^{\prime}$ and $\mathbf{b}^{\prime}$ ' state will also be caused by the hydrogen concentration difference in fused quartz. $\Delta H_{0}$ and $\Delta H^{\prime}{ }_{0}$ would be desorption energy of hydrogen from the quartz surface into vacuum of each 'channel'.

At the temperature range of over $800^{\circ} \mathrm{C}$, diffusion of hydrogen in "channels $A$ and $A$ " will be caused at the same time, and $A^{\prime}$ diffusion will dominate over $A$ with rising temperature. The following will describe why the effect of $A^{\prime}$ appears critically at a temperature of over $800^{\circ} \mathrm{C}$ only. Below $800^{\circ} \mathrm{C}$, adsorbed hydrogen molecules in a state will hop the barrier of $E_{*}$ to b. However, hydrogen in this temperature range will have not the energy to hop the barrier $E_{A}^{\prime}$ to $\mathbf{b}^{\prime}$, while, above $800^{\circ} \mathrm{C}$, it will begin for hydrogen to have energy to hop the barvier $E^{\prime}{ }_{A}$ at the same time as the quantity of hydrogen in state a decreases with rising temperature, as described above. That is, it will be found that the effect of $A^{\prime}$ dominates over that of $A$ with rising temperature above $800^{\circ} \mathrm{C}$. The relationship $E_{A}<E^{\prime}{ }_{A}$ would be required in order that hydrogen molecules on fused quartz clear the barrier $E_{A}$ only but can not clear the barrier $E^{\prime \prime}$ below $800^{\circ} \mathrm{C}$. Above $800^{\circ} \mathrm{C}$, the relationship $E^{\prime}{ }_{A}+2 \mathrm{Kcal} / \mathrm{mol} \quad\left(-\Delta H^{\prime}{ }_{1}\right)>E^{\prime} E^{\prime \prime}{ }_{A}$ would be required in order that hydrogen in $\mathbf{a}^{\prime}$ state hop the barrier $E^{\prime \prime}$, only, but does not clear barrier $E^{\prime}{ }_{A}$ back.

It is assumed that the obtained anomalous diffusion of hydrogen above $800^{\circ} \mathrm{C}$ is caused by a transition transferring of hydrogen form a channel to another.

\section{Summary ${ }^{10)}$}

It is confirmed that the diffusion coefficient of hydrogen in fused quartz falls off critically above $800^{\circ} \mathrm{C}$, and the solubility shows a sharp increase with the temperature corresponding to the anomalous diffusion. General tendencies of the solubility curves we obtained are the same as Lee's which is obtained from other direct method. Like this, we clearly recognized the diffusion phenomena of hydrogen in fused quartz in relation with the permeability and solubility at the temperature of $500^{\circ} \sim 1,100^{\circ} \mathrm{C}$.

We appreciate $\mathrm{Mr}$. Tadao Kanoh who is the chief of Lamp eng. dept. of Toshiba and translated a paper" kindly for us.

\section{Reference}

(1) J. F. Waymouth: "Electric Discharge Lamps" MIT Press, Cambridge, 257

(2) Hiroshi Washimi: J. Tllum. Engng Inst. Jpn, 59 (1975) 16

(3) V. V. Akimov, V. P. Alyavin, M. G. Andreev et al: cBe тотехника, 1 (1977) 21

(4) R. W. Lee, R. C. Frank and D. E. Swets: J. Chem. Phys., 36 (1962) 1062

(5) R. W. Lee: J. Chem. Phys., 38 (1963) 448

(6) R. W. Lee: Phys. Chem. Glasses, 5 (1964) 35

(7) R. W. Lee \& D. L. Fry: Phys. Chem. Glasses, 7 (1966) 19

(8) D. E. Swets, R. W. Lee, and R. C. Frank: J. Chem. Phys.,

(9) W. A. Rogers, R. S. Buritz, and D. Alpert: J. Appl. Phys, 25 (1954) 868

(10) Tsutomu Moriwaki, Kazuyuki Ogawa, Yoshimi Akai, and Hitoshi Imamura: J. Ill. Engng Inst. Jpn, 61, 2 (1977) 99

(11) R. Brückner: J. Non-Crystall. Solids, 5 (1971) 177

(12) T. Bell, G. Hetherington: Phys. Chem. Glasses, 3 (1962) 141 (1977.7.22, 1977.11.28) 